\title{
Reviewers for Volume 39
}

Nurul Alam, International Centre for Diarrhoeal Disease Research, Dhaka (Bangladesh)

Kirill F. Andreev, United Nations Department of Economic and Social Affairs, New York (USA)

Asaduzzaman, University of Dhaka (Bangladesh)

Roderic Beaujot, University of Western Ontario, London (Canada)

Alain Bélanger, INRS - Urbanisation, culture et société, Montreal (Canada)

Kwame Boadu, Government of Alberta, Edmonton (Canada)

K. Steve Brown, University of Waterloo (Canada)

Thomas K. Burch, University of Victoria (Canada)

Marie-Soleil Cloutier, INRS - Urbanisation, culture et société, Montreal (Canada)

Anton de Craen, Leiden University Medical Center (Netherlands)

Frank Denton, McMaster University, Hamilton (Canada)

Bertrand Desjardins, Université de Montréal (Canada)

Sven Drefahl, Stockholm University (Sweden)

Rajulton Fernando, University of Western Ontario, London (Canada)

Maxime Fougère, Human Resources and Skills Development Canada, Ottawa (Canada)

Danielle Gauvreau, Concordia University, Montreal (Canada)

Hani Guend, INRS - Urbanisation, culture et société, Montreal (Canada)

Susanne Huber, University of Veterinary Medicine, Vienna (Austria)

M. Mazharul Islam, Sultan Qaboos University, Muscat (Oman)

Charles Jones, University of Toronto (Canada)

Don Kerr, University of Western Ontario, London (Canada)

Anastasia Kostaki, Athens University of Economics and Business (Greece)

Parameswara Krishnan, University of Alberta, Edmonton (Canada)

Tracey LaPierre, University of Kansas, Lawrence (USA)

Évelyne Lapierre-Adamcyk, Université de Montréal (Canada)

Katherine Marshall, Statistics Canada, Ottawa (Canada)

Ryan Mazan, University of Western Ontario, London (Canada)

Michael E. Mercier, McMaster University, Hamilton (Canada)

Andrew Noymer, University of California, Irvine (USA)

Margaret Penning, University of Victoria (Canada)

Victor Piché, Université de Montréal (Canada)

Amélie Quesnel-Vallée, McGill University, Montreal (Canada)

K.V. Rao, India Network Foundation (USA)

James C. Raymondo, Tennessee Technological University, Cookeville (USA)

Arzu Sardarli, First Nations University of Canada, Regina (Canada)

Reto Schumacher, University of Geneva (Switzerland)

Steven J. Stack, Wayne State University, Detroit (USA)

Zongli Tang, Auburn University at Montgomery (USA)

Marc Tremblay, Université du Québec à Chicoutimi (Canada)

Bart Van de Putte, University of Ghent (Belgium)

Andrew Wister, Simon Fraser University, Vancouver (Canada)

Xuelin Zhang, Statistics Canada, Ottawa (Canada) 
“Historical Studies on Mortality," special issue, Canadian Studies in Population 39, no. 3-4 (Fall/Winter 2012) 